\title{
The Web of Data for E-Commerce in Brief
}

\author{
Martin Hepp \\ Universität der Bundeswehr München \\ E-Business and Web Science Research Group \\ Werner-Heisenberg-Weg 39, 85577 Neubiberg, Germany \\ mhepp@computer.org
}

\begin{abstract}
The GoodRelations ontology (http://purl.org/goodrelations/) is one huge success story of applying Semantic Web technology to business challenges. In this tutorial, we will (1) give a comprehensive overview and hands-on training on the conceptual structures of the GoodRelations ontology, including patterns for ownership and demand, (2) present the full tool chain for producing and consuming GoodRelations-related data, (3) explain the long-term vision of linked open commerce, (4) describe the main challenges for future research in the field, and (5) discuss advanced topics, like access control, identity and authentication (e.g. with WebID); micropayment services (like Payswarm), and data management issues from the publisher and consumer perspective.
\end{abstract}

Keywords: GoodRelations, schema.org, Semantic Web, eCommerce, eBusiness, Search Engines, RDFa, Microdata.

\section{Overview}

The GoodRelations ontology [1,2] is one of the very few OWL DL ontologies that have reached Web-scale adoption and are officially supported by major Web search engine like Google and Yahoo. GoodRelations has been implemented by major technology vendors (e.g. OpenLink Software), retailers (e.g. Bestbuy), and manufacturers (e.g. Volkswagen). In essence, GoodRelations is an industry-neutral conceptual model for representing commerce-related information that fits the needs of various stages of value chains, ranging from raw materials over manufacturing and retail to after-sales support and disposal. While GoodRelations is available in a representation based on the W3C stack for the Semantic Web vision, namely the OWL DL ontology language and the RDF data model, it can be used in arbitrary syntactical formats, including RDFa, Microdata, RDF/XML, Turtle, NTriples, dataRSS, JSON-LD, GData, or OData [3]. The GoodRelations conceptual model can be used for various purposes, namely exposing ecommerce information on the Web in a way that is easily accessible for search engines ("Semantic SEO", see [4]), browser extensions, and novel mobile applications, or for integrating product and offer information from heterogeneous sources, e.g. in data warehouses or for data quality management. 


\section{GoodRelations and the Semantic Web Vision}

One key distinction that sets GoodRelations apart from most other Web ontologies is the fact that it is stable and mature and accompanied by a comprehensive documentation and tool-chain. As a rough estimate of effort we can say that GoodRelations has so far consumed at least ten person-years of development and documentation work, and that maintaining the comprehensive documentation and supporting the GoodRelations community of adopters account for at least $75 \%$ of all effort, whereas the core ontology coding was a relatively moderate task.

GoodRelations is fully compatible with the state of the art of Semantic Web and Linked Data engineering and will work even in very sophisticated environments, e.g. where complete OWL DL reasoning is required. On the other hand GoodRelations does not critically depend on a state-of-the art Semantic Web infrastructure. In fact, GoodRelations data can be processed in any graph-based environment that follows the Entity-Attribute-Value paradigm [cf. 5].

\section{Tutorial Outline}

In this tutorial, participants will learn how to use the GoodRelations ontology to augment Web shops and other Web applications with metadata on business entities, products and services, prices, warranty, shop locations, terms and conditions, etc. This includes a comprehensive overview and hands-on training on the conceptual structures of the GoodRelations ontology including patterns for ownership and demand, an introduction to the tool-chain for producing and consuming GoodRelations-related data, and an outlook into the long-term vision of linked open commerce. We will also cover advanced topics, like access control, identity and authentication (e.g. with WebID); micropayment services (like Payswarm), and data management issues from the publisher and consumer perspective. The tutorial materials will be available from http://wiki.goodrelations-vocabulary.org/Events/ICWE2012.

Acknowledgments. The work on this paper and the ICWE 2012 tutorial have been been supported by the German Federal Ministry of Research (BMBF) by a grant under the KMU Innovativ program as part of the Intelligent Match project (FKZ 01IS10022B).

\section{References}

1. http://purl.org/goodrelations/

2. Hepp, M.: GoodRelations: An Ontology for Describing Products and Services Offers on the Web. In: Gangemi, A., Euzenat, J. (eds.) EKAW 2008. LNCS (LNAI), vol. 5268, pp. 329-346. Springer, Heidelberg (2008)

3. http://www. ebusiness-unibw. org/wiki/Syntaxes4GoodRelations

4. http://wiki.goodrelationsvocabulary.org/GoodRelations_for_Semantic_SEO

5. Dinu, V., Nadkarni, P.: Guidelines for the effective use of entity-attribute-value modeling for biomedical databases. International Journal of Medical Informatics 76(11-12), 769-779 (2007) 